\title{
Comorbidity in Specific Learning Disorder: Issues in Assessment
}

\author{
Rajshekhar Bipeta $^{1}$ \\ ${ }^{1}$ Department of Psychiatry, Institute of Mental Health, Osmania \\ Medical College, Hyderabad, India
}

\section{J Neurosci Rural Pract 2019;10:623-624}

Specific learning disorder (SLD) is a developmental disorder associated with lower than age-expected educational skills (reading, writing, and mathematics) leading to significant problems with scholastic performance. ${ }^{1}$ Childhood psychiatric disorders have high comorbidity, and SLD is no exception. The comorbidity in SLD needs to be carefully evaluated and managed, as it leads to significant functional impairment, complicates the clinical picture, and worsens the prognosis. ${ }^{2}$

In this context, in a study published in this journal ${ }^{3}$ on 7 to 12 years old children with SLD-mixed type, the investigators used Mini International Neuropsychiatric Interview for Children and Adolescents ${ }^{4}$ to diagnose psychiatric disorders and Child Behavior Checklist (CBCL) $)^{5}$ to "assess social competence and behavior problems," while, Conner's 3TM Parent Short form ${ }^{6}$ was used "to identify attention deficit/hyperactivity disorder (ADHD) and common comorbid problems." Sixty-one percent of their sample had signs of attention deficit disorder; social anxiety was found in one subject, while another child had oppositional defiant disorder and attention deficit disorder. The authors also reported difficulties in executive function, peer relations, and aggression in their sample of SLD.

For assessing learning and psychiatric disorders, when available, use of culture-fair/free and locally standardized instruments should be used. For the Indian population, National Institute of Mental Health and Neurological Sciences (NIMHANS) Index for $\mathrm{SLD}^{7}$ is standardized and recommended. ${ }^{8}$ Developmental Psychopathology Check List (DPCL) ${ }^{9}$ is a tool validated against $\mathrm{CBCL},{ }^{5}$ to screen for childhood psychopathology in the Indian setting. In school-going Indian children aged between 6 and 12 years, Bandla et $\mathrm{a}^{10}$ used NIMHANS Index ${ }^{7}$ to confirm the diagnosis of SLD and DPCL ${ }^{9}$ to assess comorbidity. The prevalence of SLD was 6.68\%, the combined (mixed) type being the most common. ADHD was the most common comorbidity (41.9\%), mostly inattentive subtype; other disorders were conduct and emotional disorders.

Altay and Görker ${ }^{11}$ reported high psychiatric comorbidity (92.5\%) in their sample of SLD cases aged between 6 and
Address for correspondence Rajshekhar Bipeta, MBBS, DPM, DNB, Institute of Mental Health, Osmania Medical College, S.R. Nagar, Hyderabad, 500038, Telangana, India (e-mail: braj111@yahoo.co.in).

15 years. The most frequent disorder was ADHD (82.3\%), followed by specific phobia, oppositional defiant disorder, enuresis, and tic disorders. Among the subtypes, the combined type of SLD (reading, writing, and math disorder) was the commonest one (37.5\%). Those with "math disorder" had lower intelligence level and higher psychiatric comorbidity.

High comorbidity (7-92\%) of SLD and ADHD in various studies $^{12}$ is a subject of particular interest and attributed to common neuropsychological and genetic risk factors. ${ }^{13}$ It remains to be seen whether other comorbidities also have similar underpinnings.

\section{Funding}

None.

\section{Conflict of Interest}

None declared.

\section{References}

1 Diagnostic and Statistical Manual of Mental Disorders (DSM-5), 5th ed. Washington, DC: American Psychiatric Association; 2013

2 Beitchman JH, Young AR. Learning disorders with a special emphasis on reading disorders: a review of the past 10 years. J Am Acad Child Adolesc Psychiatry 1997;36(8):1020-1032

3 Sahu A, Patil V, Sagar R, Bhargava R. Psychiatric comorbidities in children with specific learning disorder-mixed type: A cross-sectional study. J Neurosci Rural Pract 2019;10:617-622

4 Sheehan DV, Sheehan KH, Shytle RD, et al. Reliability and validity of the MINI International Neuropsychiatric Interview for children and adolescents (MINI-KID). J Clin Psychiatry 2010;71(3):313-326

5 Achenbach TM. Manual for the Child Behavior Checklist / 4-18 and 1991 profile. Burlington, VT: University of Vermont, Department of Psychiatry; 1991

6 Conners CK. Conners. 3rd edition: Manual. Toronto, Ontario, Canada: Multi-Health Systems; 2008

7 Hirisave U, Oommen A, Kapur M. Psychological assessment of children in the clinical setting. NIMHANS index of specific learning disabilities. Bangalore: Samudra Offset Printers; 2006
DOI https://doi.org/

10.1055/s-0039-3400694

ISSN 0976-3147.
License terms

$($ () (1) $\odot \circledast$ 
8 Shah HR, Sagar JKV, Somaiya MP, Nagpal JK. Clinical practice guidelines on assessment and management of specific learning disorders. Indian J Psychiatry 2019;61(Suppl 2):211-225

9 Hirisave U, Oommen A, Kapur M. Psychological assessment of children in the clinical setting. Developmental Psychopathology Check List for Children (DPCL) Bangalore: Samudra Offset Printers; 2006

10 Bandla S, Mandadi GD, Bhogaraju A. Specific learning disabilities and psychiatric comorbidities in school children in South India. Indian J Psychol Med 2017;39(1):76-82
11 Altay MA, Görker I. Assessment of psychiatric comorbidity and WISC-R profiles in cases diagnosed with specific learning disorder according to DSM-5 criteria. Noro Psikiyatri Arsivi 2017;55(2):127-134

12 Dupaul GJ, Stoner G. ADHD in the schools: assessment and intervention strategies. 3rd edition. New York: Guilford Press; 2014

13 Pham AV, Riviere A. Specific learning disorders and ADHD: current issues in diagnosis across clinical and educational settings. Curr Psychiatry Rep 2015;17(6):38 